\title{
The dark choroid in posterior retinal dystrophies
}

\author{
G. FISH, R. GREY, K. S. SEHMI, AND A. C. BIRD \\ From the Professorial Unit, Moorfields Eye Hospital, City Road, London EC1V 2PD
}

SUMMARY Many patients with heredomacular degeneration exhibit a peculiar fluorescein angiographic finding of absence of the normal background fluorescence (a dark choroid). The cause of this is unknown but may relate to the deposition of an abnormal material in the retinal pigment epithelial cells. The finding does not correlate with severity or duration of disease but is more frequent in patients with flecks. The finding may be useful in subdividing heredomacular degenerations into more specific disease groups.

Inherited macular degeneration comprises several different diseases of unknown aetiology, some of which are well recognised and believed to be single neurological entities, for example, Best's disease, polymorphous dystrophy, pseudoinflammatory macular dystrophy, and central areolar choroidal sclerosis. In the remainder the individual disorders are unrecognised, and attempts have been made to subdivide and catagorise them in terms of their functional, genetic, and morphological characteristics.

A peculiar fluorescein angiographic finding has been described in some of these conditions by Bonnin et al., ${ }^{1}$ who identified the absence of the dye transit during fluorescein angiography. Other authors have illustrated this finding but have not commented on it. ${ }^{23}$ The cause and significance of this phenomenon are unknown. This retrospective study was undertaken to identify the morphological features of the 'dark choroid' and to assess its clinical significance.

\section{Patients and methods}

The diagnostic files from the Retinal Diagnostic Department at Moorfields Eye Hospital were used to retrieve the records of patients believed to have inherited macular dystrophy in which serous retinal detachment did not occur, including those classified as Stargardt's disease, bull's eye maculopathy, cone dystrophy, butterfly dystrophy, and cone rod dystrophy. Best's disease, Sorsby's pseudoinflammatory macular dystrophy, posterior polymorphous dystrophy, dominant drusen, patients with onset of

\footnotetext{
Correspondence to Professor A. C. Bird.
}

visual loss after 60 years of age, and those with degeneration due to toxic agents were excluded.

Stereo colour fundus photographs and stereo angiography were accomplished on all patients, and as many as possible had electrodiagnostic studies and testing of visual fields and colour vision.

Colour vision testing included HRR plates, Ishihara places, panel D 15, and Farnsworth-Munsell 100 hue. Visual fields were recorded on a Goldmann perimeter, and electrodiagnostic tests included electro-oculography (EOG), photopic and scotopic electroretinography (ERG), and flicker-fusion measurements.

Fluorescein angiography was performed with 4-5 $\mathrm{ml}$ of a $20 \%$ fluorescein solution injected intravenously in the antecubital vein, and photographs were taken with the Zeiss fundus camera and Ilford PP4 film.

\section{Results (Table 1)}

A total of 91 patients were included in this study. A dark choroid was identified on an angiogram in which the choroidal fluorescence was undetected throughout the study (Fig. 1). This was most easily identified during the dye transit period. A majority of the patients had either obviously dark or normal choroids, but some variation was encountered. In several patients with widespread pigment epithelial disease in the posterior pole lack of choroidal pattern could be identified in the peripheral $\left(10^{\circ}\right.$ to $15^{\circ}$ ) part of the posterior pole or in the immediate peripapillary region (Fig. 2). Occasionally a patient with central pigment epithelial disease had a dark ring round the site of maximum abnormality, but the peripheral choroid appeared normal. These 
patients were classified as having normal choroids.

Of the 91 patients in this study $37(41 \%)$ had a dark choroid, and in the remainder the background choroidal pattern was well seen (Fig. 3).

\section{ASSOCIATED CLINICAL ATTRIBUTES}

Morphology of disease. Flecks: Forty-seven patients

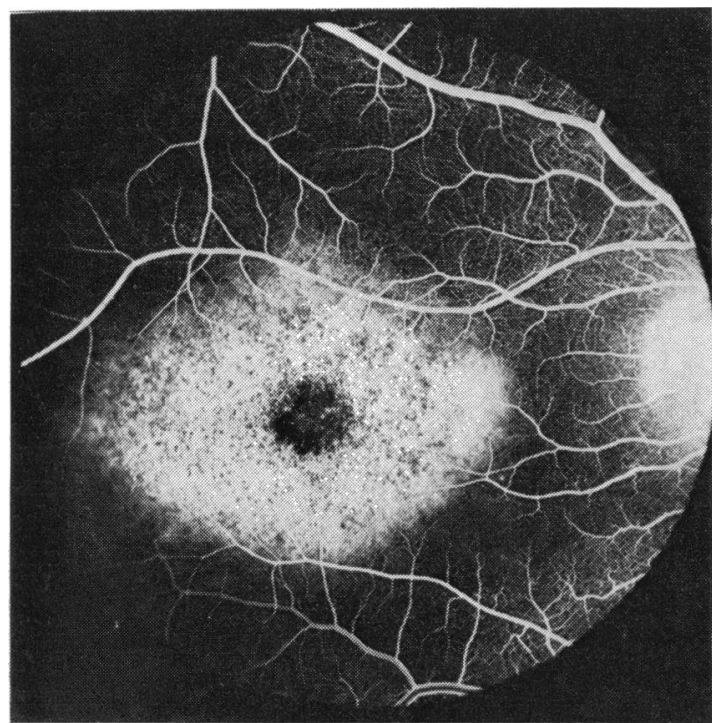

Fig. 1 Fluorescein angiogram of a patient with bull's eye dystrophy showing the dark choroid and retinal capillaries.

Table 1 Clinical details of patients within the study

\begin{tabular}{|c|c|c|c|c|c|}
\hline & & & $\begin{array}{l}\text { Dark } \\
\text { choroid }\end{array}$ & Normal & Total \\
\hline \multirow{3}{*}{ Severity } & $\begin{array}{l}\text { Abnormal } \\
\text { ERG }\end{array}$ & $\begin{array}{l}\text { CFF } \\
\text { Scoptic }\end{array}$ & $\begin{array}{l}3 / 17 \\
2 / 23\end{array}$ & $\begin{array}{l}3 / 33 \\
8 / 39\end{array}$ & $\begin{array}{l}6 / 50 \\
10 / 62\end{array}$ \\
\hline & & $\begin{array}{l}\text { Photopic } \\
\text { Abnormal } \\
\text { EOG }\end{array}$ & $\begin{array}{l}8 / 23 \\
16 / 22\end{array}$ & $\begin{array}{l}18 / 39 \\
24 / 37\end{array}$ & $\begin{array}{l}26 / 62 \\
40 / 59\end{array}$ \\
\hline & & $\begin{array}{l}\text { Duration } \\
\text { Age onset before } \\
15 \text { years old }\end{array}$ & $\begin{array}{l}14 / 33 \\
12 / 32\end{array}$ & $\begin{array}{l}17 / 41 \\
13 / 42\end{array}$ & $\begin{array}{l}31 / 74 \\
25 / 74\end{array}$ \\
\hline Vision & & $\begin{array}{l}6 / 36 \text { or worse } \\
6 / 18 \text { or better } \\
\text { Bull's Eye }\end{array}$ & $\begin{array}{l}6 / 36 \\
9 / 37 \\
6\end{array}$ & $\begin{array}{l}12 / 52 \\
18 / 54 \\
12\end{array}$ & $\begin{array}{l}18 / 88 \\
27 / 91 \\
18\end{array}$ \\
\hline Morphology & & $\begin{array}{l}\text { Flecks } \\
\text { Female } \\
\text { Male } \\
\text { Sporadic } \\
\text { (patients) }\end{array}$ & $\begin{array}{l}34 \\
15 \\
22 \\
22\end{array}$ & $\begin{array}{l}13 \\
22 \\
32 \\
31\end{array}$ & $\begin{array}{l}47 \\
37 \\
54 \\
53\end{array}$ \\
\hline \multirow[t]{2}{*}{ Heredity } & & $\begin{array}{l}\text { Recessive } \\
\text { (families) }\end{array}$ & 6 & 3 & 9 \\
\hline & & $\begin{array}{l}\text { Dominant } \\
\text { (families) }\end{array}$ & & 10 & 10 \\
\hline \multicolumn{3}{|c|}{ Total Patients } & 37 & 54 & 91 \\
\hline
\end{tabular}

$\mathbf{C F F}=$ critical flicker fusion frequencs . were identified as having white flecks at the level of the pigment epithelium. Of the 37 with dark choroids 34 had flecks $(92 \%)$, whereas of the 54 patients with a normal choroidal pattern only $13(24 \%)$ had flecks. The 3 patients with dark choroids but no flecks had a bull's eye pattern of pigment epithelial disease. In patients with identifiable choroidal fluorescence

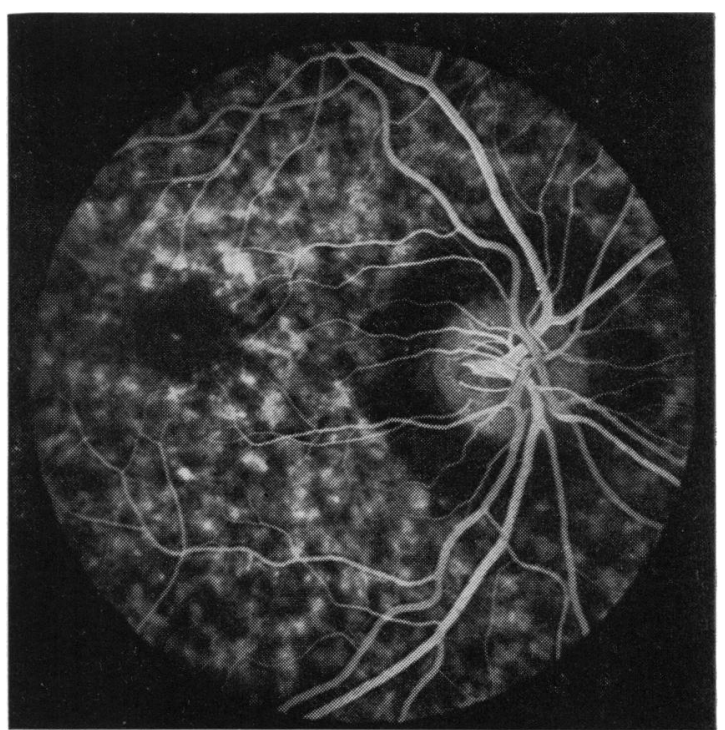

Fig. 2 Fluorescein angiogram of patient with Stargardt's disease showing hypofluorescence in the peripapillary region.

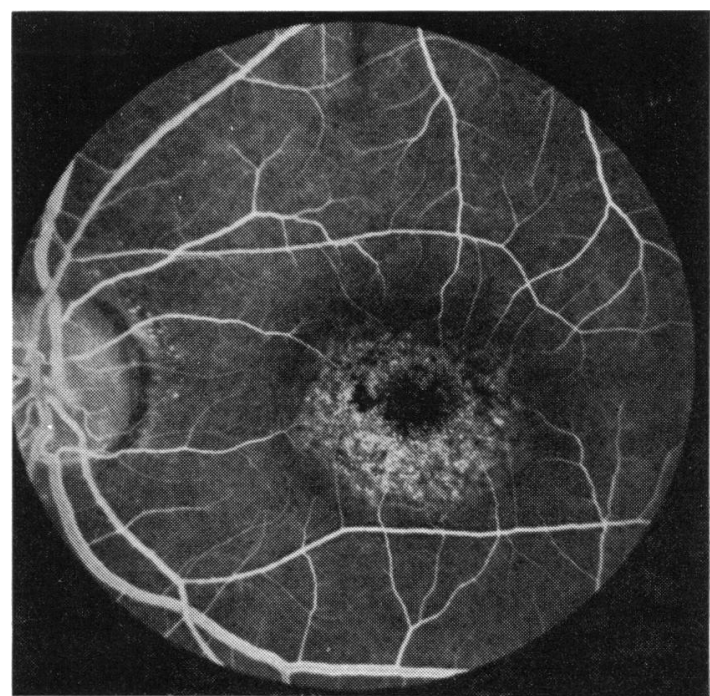

Fig. 3 Fluorescein angiogram of a patient with bull's eye dystrophy and light choroid. 
on angiography the white flecks corresponded with areas of hypofluorescence in the angiogram, and there was hyperfluorescence corresponding with areas of pigment epithelial atrophy. In patients with dark choroids the centre of the flecks appeared dark on the angiogram but was usually surrounded by a halo of hyperfluorescence.

Retinal capillary changes: In most patients with dark choroids, the retinal vascular pattern could be seen easily, including minute details of the retinal capillaries. In many patients the capillaries appeared unusually prominent, as if the whole capillary bed were dilated (Fig. 1). In no patient was dye leakage seen from these capillaries into the neuroretina.

Distribution of retinal pigment epithelial changes: Five morphological patterns of pigment epithelial disease were seen in these patients with atrophic heredomacular degeneration. (1) Bull's eye lesions: A bull's eye lesion consisted of a relatively normal foveola surrounded by a ring of pigment epithelial atrophy. Of the 18 patients with bull's eye dystrophies 6 had a dark choroid. The dark foveola was surrounded by a fluorescent ring consisting of transmission defects surrounded by darkness of the remainder of the posterior pole (Fig. 1). The 12 remaining patients had a normal choroidal pattern associated with bull's eye dystrophy (Fig. 3). (2) Atrophic foveal lesions with flecks: 31 patients had varying degrees of atrophy of the central pigment epithelium associated with multifocal pigment epithelial atrophy scattered elsewhere in the posterior pole and white flecks at the level of the pigment epithelium. Twenty-one of these patients had dark choroids and 10 had normal choroids. In all cases the area of pigment epithelial atrophy was hyperfluorescent on angiography, whether the choroid was dark or light. (3) Atrophic macular lesions without accompanying flecks: There were 18 such patients and all had a normal choroidal pattern. (4) Large posterior pole lesions: Such patients had a large central area of retinal pigment epithelial atrophy associated with varying degrees of choriocapillaris atrophy. There were 19 such patients, of whom 10 had dark choroids (all having flecks), and the remaining 9 had a normal choroidal pattern ( 2 having flecks). (5) The last 5 patients had small dots at the level of the pigment epithelium associated with variable atrophy of the macula. All had normal choroid on fluorescein angiography.

Fluorescein angiograms on patients with Best's disease, central areolar choroidal sclerosis, Sorsby's pseudoinflammatory macular dystrophy, posterior polymorphous dystrophy and chloroquine toxicity, and dominant drusen were examined systematically, and in none of these patients was a dark choroid identified.
Inheritance. Dominant: Of the 10 families seen with dominantly inherited macular dystrophy all members had normal appearing choroids on fluorescein angiography. Recessive: Of the 9 patients with recessive disease, 6 had a dark choroid $(67 \%)$ and the remainder had normal choroids (1 patient had a dark choroid but his brother had a normal choroidal filling pattern). Sporadic: Of the 53 patients with sporadic disease, 22 had dark choroids $(41 \%)$ and the remainder had normal choroids. No patient was identified as having $\mathrm{X}$-linked disease.

Sex. Fifteen of 37 females $(41 \%)$ and 22 out of 54 males $(41 \%)$ had dark choroids.

Severity. The visual acuity was $6 / 18$ or better in both eyes of 9 patients with dark choroid $(25 \%)$ and 18 with light choroid $(33 \%)$.

Age of onset of disease. Twelve of 32 patients with dark choroids had onset before age $15(36 \%)$ compared with 13 of 42 patients with normal choroid (31\%).

Duration of disease. Fourteen of 33 patients with dark choroids had symptoms for less than 5 years $(42 \%)$ as opposed to 17 of 41 patients having normal choroids $(41 \%)$.

Colour vision. Most of the patients had poor colour discrimination without any particular pattern of colour visual loss, such that no useful information could be derived from this test.

Visual fields. All patients who were tested had a central or paracentral field loss. No correlation between the pattern of field loss and the choroidal appearance was identified.

Electro-oculography. Of 59 patients on whom electro-oculography was undertaken 40 had a reduced EOG (less than $180 \%$ light-induced rise in ocular potential). Sixteen out of 22 patients with dark choroids had decreased EOG (73\%) compared with 24 of 37 patients with normal choroids $(65 \%)$.

Electroretinography. Of the 62 patients who had electroretinography 26 had abnormal photopic ERGs. Of the 23 patients with dark choroids 8 had reduced potentials $(35 \%)$ compared with 18 of 39 $(46 \%)$ in which the choroid was normal. Ten of the 62 patients had abnormal scoptic ERGs. Of the 23 with dark choroids 2 were abnormal $(9 \%)$, and of the 39 with normal choroids, 8 had subnormal scoptic potential $(20 \%)$. Flicker fusion was undertaken in 50 patients, and in $6(12 \%)$ the critical flicker fusion frequency was less than 30 cycles per second. Of the 17 with dark choroids 3 had abnormal critical flicker fusion ( $18 \%$ ) compared with 3 of the 33 patients $(9 \%)$ with normal choroids.

\section{Discussion}

It could be argued that the dark choroid does not represent a true morphological entity. There is no 
doubt that an underexposed or underdeveloped negative or overcontrasted print may create this appearance artefactually. However, many of our patients had 2 or more studies undertaken, and this fluorescein angiographic finding is quite repeatable. Had it been a photographic phenomenon, dark choroids would have been seen in other conditions, yet it was not identified in any patients with Best's disease, polymorphous dystrophy, or pseudoinflammatory dystrophy during a systematic search. These considerations lead us to believe that the dark choroid is an angiographic sign likely to be related to specific pathological changes in these patients, and may be related to the pathogenesis of disease.

Bonnin et al. ${ }^{1}$ considered 2 possible causes for the nonvisualisation of the choroid in these patients. Firstly, they thought it possible that an abnormal deposit of material existed at the level of the outer retina, pigment epithelium, or Bruch's membrane, which absorbed visible light of short wavelengths, so preventing illumination of the choroid during fluorescein angiography. He dismissed this explanation as unlikely, since he argued that such a layer would be seen by ophthalmoscopy. The alternative explanation considered was nonfilling of the choroid, and Bonnin et al. favoured this as the cause of this angiographic sign. It is hard to imagine that the patient studied by us had no choroidal circulation, since greater outer retinal dysfunction might have been expected in the presence of total choroidal ischaemia. A greater objection to this theory is the fact that the hyperfluorescence of choroidal origin was seen at the sites of manifest pigment epithelial abnormalities and maximum receptor disease whether they were confluent as in bull's eye dystrophy or multifocal as in Stargardt's disease.

Therefore it is worth while reconsidering the first possibility, the rejection of which may not be fully justified. If a layer of abnormal material were spread evenly over the whole fundus in the deeper retinal layers, it would not necessarily alter the appearance when viewed with white light. Relatively little blue-green light is reflected from choroid normally, ${ }_{4}^{4}$ and an additional blue-green filter would have little noticeable effect. The presence of an abnormal absorbing layer is not inconsistent with current concepts of retinal receptor disease; deposits occur at the level of the pigment epithelium in a localised fashion in Best's disease and in Stargardt's disease ${ }^{5}$ and diffusely in the Royal College of Surgeons of England rats. ${ }^{7}$

Furthermore, if the integrity of this abnormal layer were dependent upon constant receptor turnover, as is the case in RCS rats, it would disappear at sites of maximum receptor disease and create relative hyperfluorescence. This correlates precisely with clinical observations. These considerations suggest that this angiographic sign is due to deposition of abnormal blue-green-absorbing material at the level of or near to the pigment epithelium. This view is reinforced by the observation that flecks are present in nearly all patients with dark choroids since these flecks may represent areas of local concentration of this abnormal material.

Apparent enlargement of the retinal capillaries was commonly associated with dark choroids. It is possible that this may be a photographic phenomenon, whereby these vessels are silhouetted against the dark background, but we agree with previous authors ${ }^{3}$ that this is likely to represent true dilatation, since the changes are so marked. It is possible that the dark choroids and retinal vessel changes are pathogenically related. If the abnormal layer caused or was due to an abnormality of metabolic exchange between the choroid and outer retina, it would predictably influence the retinal vasculature.

Evidence was sought to assess the likelihood that dark choroids indicated the presence of a specific pathological process present in some cases but absent in others, or as to whether it occurred in all cases at some stage in the evolution of the disease. Our observations do not indicate that this angiographic sign occurs consistently in either early or late disease, and equally it is not associated with severe or mild disease. That a dark choroid has not yet been identified in families with autosomal dominant macular degeneration supports to some extent the hypothesis that the dark choroid is a clinical characteristic of certain specific central receptor dystrophies. However, the presence of a dark choroid in only one of 2 affected siblings casts some doubt on the validity of the concept that a dark choroid implies a specific metabolic abnormality.

If this hypothesis were correct, the presence or absence of a dark choroid might provide a means of subdividing autosomal recessive macular degenerations, such as Stargardt's and bull's eye dystrophies, into more specific disease groups. Also, it may give some clue to the nature of the pathological processes taking place in these conditions, namely, that the disease causes deposition of abnormal material in the outer retina or inner choroid, as a long-standing change over the whole of the posterior fundus. This concept is supported by recent observations of histopathological changes in bull's eye dystrophy. ${ }^{\text {? }}$ Such material does not itself appear to interfere significantly with retinal function and does not presage rapid loss of vision in the affected area.

\section{References}

1 Bonnin P, Passot M, Triolaire M Th. Le signe du silence choroidien dans les degenerescences tapeto-retiennes 
posterieures. In: De Laey, JJ, ed. Internation Symposium on Fluorescein Angiography. Doc Ophthalmol 1976; 9: 461-3 Proc Ser.

2 Krill AE. In: Krill AE, ed. Flecked Retinal Diseases in Hereditary Retinal and Choroidal Diseases. New York: Harper and Row, 1977; 2: 768-77.

3 Notting JGA, Deutman AF. Leakage from retinal capillaries in hereditary dystrophies. In: De Laey JJ, ed. International Symposium on Fluorescein Angiography. Doc Ophthalmol 1976; 9: 439-47. (Proc. Ser.).

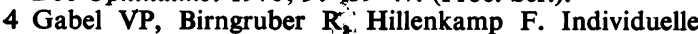
Unterschiede der Lichtabsorption am Augenhintergrund in Sichtbaren und infraroten Spectralbereich. Deutsche Ophthalmologische Gessellschaft. Berichtuberdie 1977; 74 (Zusammenkunft): 418-21.

5 Klein BA, Krill AE. Fundus flavimaculatus: clinical, functional and histopathological observations. Am J Ophthalmol 1967; 64: 3-23.

6 Dowling JE, Sidman RL. Inherited retinal dystrophy in the rat. J Cell Biol 1962; 14: 73-109.

7 Eagle RC, Lucier RC, Bernardino VB, Yanoff M. Retinal pigment epithelial abnormalities in fundus flavimaculatus -a light and electron microscopic study. Ophthalmology $1980 ; 87: 1189-200$. 